\title{
Brain Abscess Caused by Nocardia: Case Report and Literature Review
}

\section{Abscesso Cerebral causado por Nocardia: Relato de Caso e Revisāo da Literatura}

\author{
Guilherme Finger ${ }^{1}$ Maria Eduarda Conte Gripa ${ }^{2}$ Tiago Paczko Bozko Cecchini2 ${ }^{2}$ \\ Tobias Ludwig do Nascimento ${ }^{10}$ \\ ${ }^{1}$ Neurosurgery Department, Hospital Cristo Redentor, Porto Alegre, RS, Brazil \\ 2 Universidade Luterana do Brasil, Canoas, RS, Brazil \\ Address for correspondence Guilherme Finger, MD, Rua Domingos \\ Rubbo, 20, Porto Alegre, RS, 91040-000, Brazil \\ (e-mail: guilhermefingermd@gmail.com). \\ Arq Bras Neurocir 2021;40(3):e280-e283.
}

\begin{abstract}
Keywords

- brain abscess

- nocardia

- infection

Resumo

Palavras-chave

- abscesso cerebral

- nocardia

- infecçāo

Nocardia brain abscess is a rare clinical entity, accounting for $2 \%$ of all brain abscesses, associated with high morbidity and a mortality rate 3 times higher than brain abscesses caused by other bacteria. Proper investigation and treatment, characterized by a longterm antibiotic therapy, play an important role on the outcome of the patient. The authors describe a case of a patient without neurological comorbidities who developed clinical signs of right occipital lobe impairment and seizures, whose investigation demonstrated brain abscess caused by Nocardia spp. The patient was treated surgically followed by antibiotic therapy with a great outcome after 1 year of follow-up.

Abscesso cerebral por Nocardia é uma entidade rara, correspondendo a $2 \%$ dos abscessos cerebrais. Quando comparada a abscessos causados por outras bactérias, apresenta morbimortalidade 3 vezes maior. Adequada investigação e tratamento, caracterizado por antibioticoterapia prolongada, são cruciais para desfecho favorável dos pacientes. Os autores descrevem um caso de uma paciente sem comorbidades neurológicas prévias que apresentou sinais de comprometimento do lobo occipital direito e crises convulsivas, cuja investigação demonstrou tratar-se de abscesso cerebral por Nocardia spp. A paciente foi tratada cirurgicamente seguida de antibioticoterapia prolongada, com ótima evolução ao longo de 1 ano de acompanhamento.
\end{abstract}

\section{Introduction}

Nocardia is a genus of the Nocardiaceae family, described by Edmon Nocard as aerobic filamentous Gram-positive, weak-acid bacteria. Nocardia species live worldwide as soil saprophytes specially in organic detritus area, and include Nocardia farcinica, Nocardia brasiliensis, Nocardia transvalensis and Nocardia otitidiscaviarum. ${ }^{1,2}$ However, the species most associated with human infection is Nocardia asteroides. ${ }^{3}$ Although nocardia brain abscess is a rare clinical entity, accounting for only $2 \%$ of all brain abscesses, it is associated with high morbidity, and the mortality is 3 times higher than brain abscesses caused by other bacteria. ${ }^{4,5}$ Mortality rate is even higher in patients with multiple abscesses or with immunosuppressive conditions. ${ }^{5,6}$

Nocardia is considered an opportunistic infection, associated with defects in cell-mediated immunity. ${ }^{3}$ Risk factors for this condition include male, autoimmune diseases, alcohol abuse, innutrition, chronic pulmonary disease and all the received

August 25, 2020

accepted

September 25, 2020

published online

November 26, 2020
DOI https://doi.org/ 10.1055/s-0040-1719124. ISSN 0103-5355.

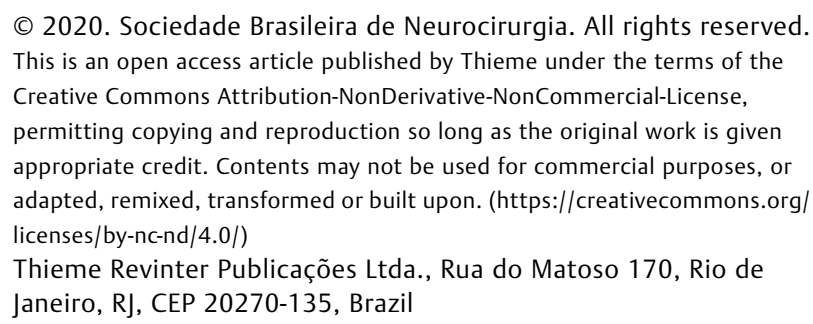


situations that affect the immune system, such as HIV infection, transplantation, cancer (mainly hematological) and longterm corticosteroid use., ${ }^{1,3}$ Patients who have received solidorgan transplants are also at higher risk. ${ }^{7}$

The pathogen is not transmitted personally, it may be inhaled through the respiratory tract, ${ }^{1}$ especially on dust particles, or may be directly inoculated into the skin or subcutaneous tissues. Subsequent hematogenous dissemination may lead to infection in any organ, with a particular predilection for the central nervous system (CNS). ${ }^{1,5}$

The development and severity of the disease depend on the interaction between the bacteria (pathological invasive potential) and the immune system of the host. There are 6 different forms of nocardial infection disease: pulmonary nocardiosis; systemic nocardiosis (involving two or more body sites); CNS nocardiosis; extrapulmonary nocardiosis; cutaneous and actinomycetoma. ${ }^{2}$ Cerebral nocardiosis constitutes the most severe form of infection; it can be either an isolated lesion, without evidence of extracranial involvement, or be part of a disseminated disease. Current data states that $71 \%$ of nocardial infections affecting the CNS are part of a disseminated infection, specially associated with pulmonary or cutaneous diseases., ${ }^{8,9}$

The authors present a case report of a patient who evolved with visual deficits and seizure, whose investigation diagnosed a brain abscess secondary to Nocardia spp. The patient was submitted to surgical resection of the lesion and prolonged antibiotic therapy with a great outcome after 1 year of follow-up.

\section{Case Report}

A 67-year-old female was admitted at the emergency department after fainting at home. The patient had no personal nor family history of neurological diseases or seizures. During hospital admission, the patient was confused but had no signs of motor or sensitive deficits, no aphasia nor dysarthria, superficial and deep reflexes were normal and symmetrical, there was no signs of cerebellar or brainstem compromise. The patient had no fever and there was no sign of neck stiffness. After 30 minutes of hospital admission, the patient described visual alteration that was followed by a seizure that was promptly controlled after $10 \mathrm{mg}$ of diazepam infusion. A brain computed tomography (CT) was performed, demonstrating a lesion in the right occipital lobe with vasogenic edema, whose lesion had a peripheric enhancement after contrast infusion (-Fig. 1). The patient was transferred to the neurosurgery department, whose neurological exam evidenced a left homonymous hemianopsia, with no other deficits. The investigation was complemented with a magnetic resonance (MRI), which demonstrated a right occipital lobe lesion measuring $36 \mathrm{~mm}$ anteroposteriorly, $24 \mathrm{~mm}$ laterolaterally and $31 \mathrm{~mm}$ craniocaudally. On T1 weighted images, the lesion was hypointense with a circular enhancement after gadolinium infusion; fluid-attenuated inversion recovery (FLAIR) and T2 weighted images demonstrated a vasogenic edema surrounding the lesion, and diffusion images demonstrated an important restriction inside the lesion. The characteristics presented in the MRI suggested a case of brain abscess (-Fig. 2).

The patient was submitted to blood tests (including blood culture), chest X-ray and pulmonary angiotomography, but all exams were normal (no bacteria in the blood culture samples were identified, no signs of arteriovenous shunts in the lungs or heart. The medical history of the patient included hypertension, controlled with losartan and anlodipin.

A neurosurgical procedure was performed, with a right occipital craniotomy, aspiration of the lesion content (which was sent for Gram analysis and culture) and the capsule was removed. The patient presented a good recovery after surgery, remaining with the left homonymous hemianopsia, but no other neurological deficits.

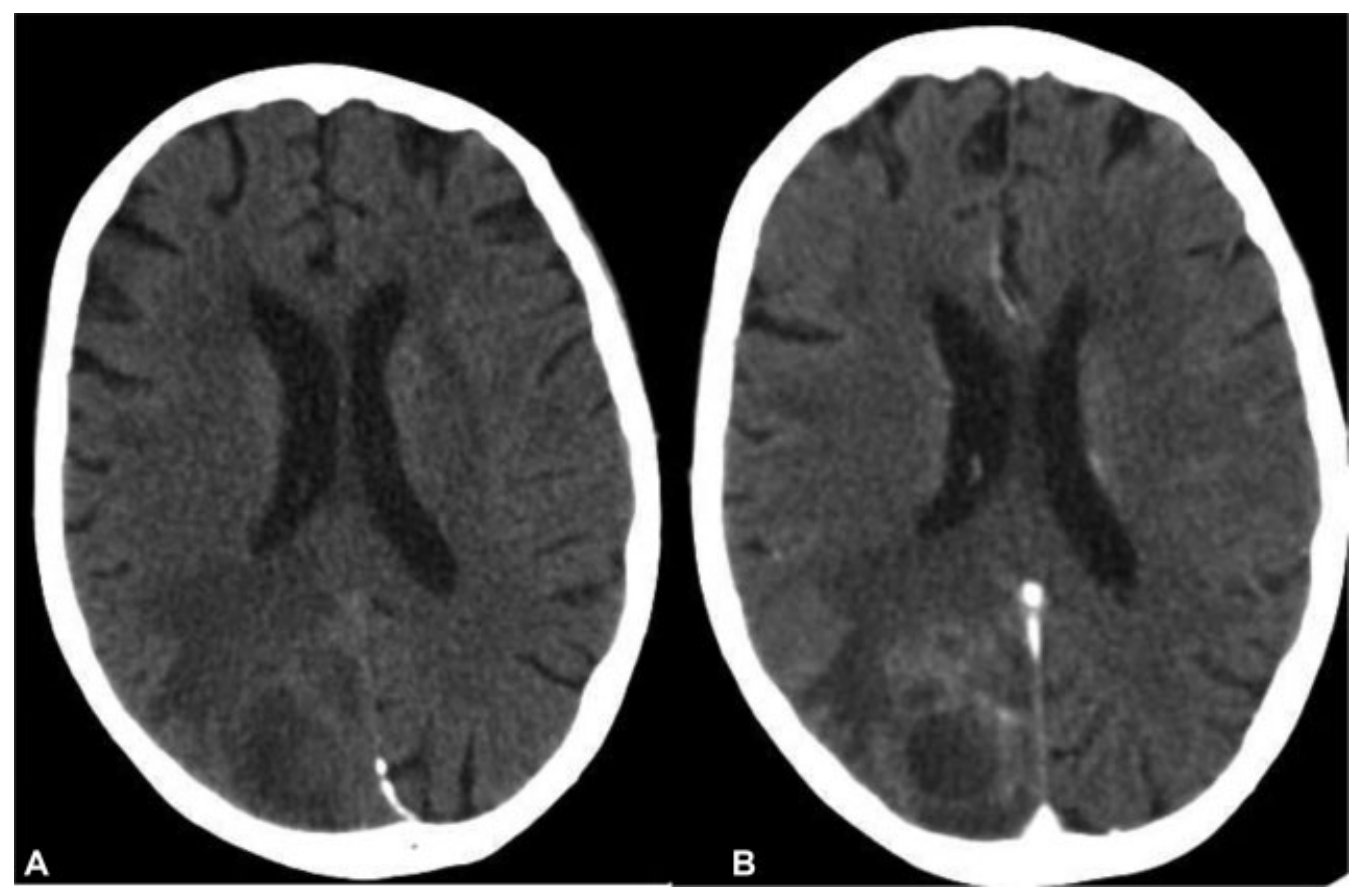

Fig. 1 Computed tomography images. Computed tomography in axial images, without contrast infusion (A) and after contrast infusion (B). 


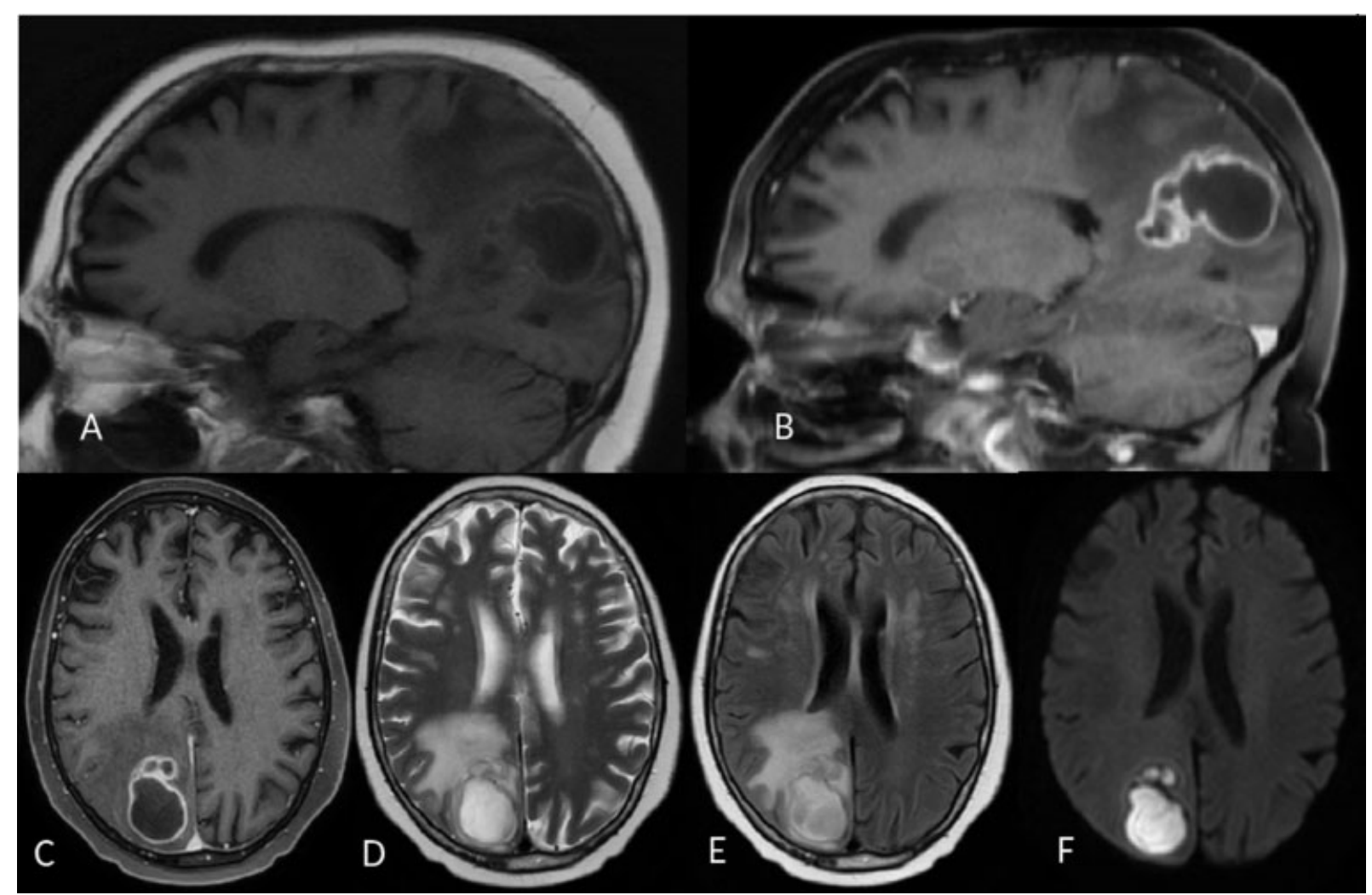

Fig. 2 Magnetic resonance images. Magnetic resonance images on sagittal plain without gadolinium infusion (A) and after gadolinium infusion with a peripheral enhancement (B). Axial imagens in T1 weighted with gadolinium (C), T2 weighted (D), FLAIR (E) and diffusion (F).

The Gram stain did not demonstrate any pathogens; however, the culture identified the growth of Nocardia spp. The patient was treated in the hospital with trimethoprim/ sulfamethoxazole and imipenem during 4 weeks, presenting a good neurological and clinical recovery (remaining only with the left homonymous hemianopsia), being discharged under the treatment of trimethoprim/sulfamethoxazole twice a day for 1 year. During the follow-up, the patient had no complaints, with no neurological nor systemic compromise. The cerebral computed tomography (CT) scan performed after 1 year of follow-up showed no signs of residual abscess and the patient was released back to primary care.

\section{Discussion}

Unlike primary pulmonary and cutaneous infections, which may be self-limiting, lesions of disseminated nocardiosis progress unless treated. ${ }^{3}$ Central nervous system abscess can be isolated or multiple; the number of abscesses and their location will clinically determine the neurological manifestation. ${ }^{1}$ Solitary abscess is the most common finding; however, multiple lesions are not uncommon, being reported in 38\% of the cases. ${ }^{10}$

During investigation, lumbar puncture is not part of diagnosis procedures for brain abscess, and should be performed only when there is clinical suspicion of meningitis or abscess rupture into the ventricular system and there is no contraindications for lumbar puncture. ${ }^{7}$

The diagnosis of brain abscess can be suggested by image exams, especially MRI. On MRI, pyogenic brain abscess is characterized by a hypointense lesion surrounded by a capsule that enhances after gadolinium infusion on T1 weighted series; diffusion-weighted series show a hyperintense signal within the abscess and hypointense on apparent-diffusion-coefficient imaging. ${ }^{7}$ Diffusion-weighted imaging has a sensitivity and specificity of $96 \%$ to differentiate brain abscess and tumor, with a positive predictive value of $98 \%$ and negative predictive value of $92 \%{ }^{11}$ Nocardial brain abscess has similar features of a pyogenic abscess on MRI.

When the physician has suspicion of brain abscess, it is reasonable to postpone the therapy until the neurosurgical procedure is performed, as long as the patient is clinically stable and the surgery can be performed soon. If the patient has systemic disease (pulmonary or skin, for example), the sample can be retrieved from any of those places. If Nocardia spp. is identified and the patient is clinically stable with brain lesion $<2 \mathrm{~cm}$, the neurosurgeon is authorized to indicate antibiotic therapy alone. ${ }^{10}$ Under this therapy, stereotactic aspiration is recommend if the clinical condition deteriorates or if the lesion does not decrease within 1 month of antibiotic treatment. $^{12}$

In patients with CNS nocardiosis, the treatment protocol depends on the condition of the immune system of the patient. Immunocompetent patients presenting one of the following situations: intracranial abscess $>2.5 \mathrm{~cm}$, multiple abscesses, cerebellum or posterior fossa involvement, lesion growth after 2 weeks of antibiotic treatment, failure to reduce size within 1 month, should undergo a neurosurgical resection. ${ }^{8}$ Immunocompetent patients without those criteria may be submitted to stereotactic biopsy and wait for the identification of the pathogen, which facilitates an early and accurate diagnosis with an 
antimicrobial sensitivity profile specific to the intracranial lesion. ${ }^{12}$

Immunocompromised patients should always be submitted to neurosurgical procedure, because they are more likely to have atypical infections and neoplasms, multiple intracranial lesions and a higher mortality rate. ${ }^{12,13}$

Nocardia spp. usually grows in regular means of bacteriological culture. However, cultural diagnosis requires a longer period for bacterial isolation, once it is a slow-growing pathogen and the laboratory is often unable to identify the microorganism using routine techniques. Therefore, the laboratory should be notified about the suspicion of Nocardia spp. infection, so the culture will be accompanied for a longer period. 1,5,6

Because of its excellent CNS penetration, trimethoprimsulfamethoxazole remains the first line treatment, except in patients with known hypersensitivity to it. ${ }^{12}$ The prescription of trimethoprim-sulfamethoxazole in high parenteral doses (15 mg/kg trimethoprim and $75 \mathrm{mg} / \mathrm{kg}$ sulphamethoxazole daily) is recommended for 6 weeks, after which the therapy can be reduced or changed depending on the clinical and radiological responses, followed by oral therapy. Central nervous system nocardiosis should be treated for a total of 12 months ${ }^{5}$ and monitored for at least a further year after completion of treatment. ${ }^{8}$ Herrero Martínez et al. recommend lifetime secondary prophylaxis for patients with unrecoverable immunocompromised states. ${ }^{1}$

Nocardia spp. is generally resistant to penicillin, ${ }^{6}$ and second line agents include minocycline, imipenem, and third-generation cephalosporins combined with aminoglycoside. ${ }^{14,15}$

The mortality rate is $20 \%$ for immunocompetent and $55 \%$ for immunocompromised patients. ${ }^{10}$ The recognized factors leading to a good outcome are: early diagnosis, institution of appropriate antimicrobial therapy, lack of underlying systemic disease, and limited disease caused by Nocardia spp. ${ }^{12}$

The present article has some weakness that must be stated. First, it is a case report, which, in a scientific point of view, does not have the same power of evidence as other most sophisticated study designs. Second, the incidence of primary brain abscess has diminished in the last decades; therefore, Nocardia brain abscess (which represents $2 \%$ of all brain abscesses) will not be expected to be frequent among neurosurgical cases. Third, the investigation algorithm of brain abscess remains the same, and should not be changed to try to identify a bacterium that accounts for $<5 \%$ of cases.

However, the article has some strengths that deserve to be highlighted. The literature review presented embraces important information about Nocardia (including biological characteristics, which are usually unknow by doctors) and the different types of human affection by this bacterium, demonstrating that brain abscess is one of the types of human infection by Nocardia spp. Also, the discussion brings information about proper investigation and therapy of brain abscesses in general, describing when antibiotic therapy should be promptly prescribed or when a surgical procedure is required prior to the antibiotic therapy. Finally, the case report and the review have two main notes. The first one is that brain abscess caused by Nocardia spp. is curable and can be treated with a single antibiotic(trimethoprim-sulfamethoxazole). The second signif- icant information is that proper treatment requires a long-term therapy with antibiotic, which must be maintained for, at least, 1 year; or during the lifetime in immunocompromised patients.

\section{Conclusion}

Even though the incidence of brain abscess due to Nocardia spp. is rare and have a higher morbidity and mortality when compared with other pyogenic abscesses, its diagnosis and optimized surgical and antibiotic therapy may cure the patient without neurological deficits.

Note

The patient has consented to the submission of the case report to the journal.

Conflict of Interests

The authors have no conflict of interests to declare.

\section{References}

1 Herrero Martínez JA, Gómez Gómez J, García Vázquez E, Hernández Torres A. Nocardiosis. Med - Programa Form Médica Continuada Acreditado [Internet]. 2014;11(58):3412-3418. Available from: http://linkinghub.elsevier.com/retrieve/pii/S0304541214707930

2 Beaman BL, Beaman L. Nocardia species: host-parasite relationships. Clin Microbiol Rev 1994;7(02):213-264

3 Lerner PI. Nocardiosis. Clin Infect Dis 1996;22(06):891-903, quiz 904-905

4 Tamarit M, Poveda P, Barón M, Del Pozo JM. Four cases of nocardial brain abscess. Surg Neurol Int 2012;3:88http://www.ncbi.nlm. nih.gov/pmc/articles/PMC3463145/

5 Kennedy KJ, Chung KHC, Bowden FJ, et al. A cluster of nocardial brain abscesses. Surg Neurol 2007;68(01):43-49, discussion 49

6 Yaakup NA, Rahmat K, Ramli N, Sia SF. Cerebral nocardiosis in an immunocompetent patient: A diagnostic dilemma. Eur J Radiol Extra 2009;71(02):47-51

7 Brouwer MC, Tunkel AR, McKhann GM II, van de Beek D. Brain abscess. N Engl J Med 2014;371(05):447-456http://www.nejm. org/doi/abs/10.1056/NEJMra1301635

8 Kilincer C, Hamamcioglu MK, Simsek O, et al. Nocardial brain abscess: review of clinical management. J Clin Neurosci 2006;13 (04):481-485http://www.ncbi.nlm.nih.gov/entrez/query.fcgi? cmd=Retrieve\&db=PubMed\&dopt=Citation\&list_uids=16678731

9 Malincarne L, Marroni M, Farina C, et al. Primary brain abscess with Nocardia farcinica in an immunocompetent patient. Clin Neurol Neurosurg 2002;104(02):132-135

10 Mamelak AN, Obana WG, Flaherty JF, Rosenblum ML, Holtom PD. Nocardial brain abscess: treatment strategies and factors influencing outcome. Neurosurgery 1994;35(04):622-631

11 Reddy JS, Mishra AM, Behari S, et al. The role of diffusionweighted imaging in the differential diagnosis of intracranial cystic mass lesions: a report of 147 lesions. Surg Neurol 2006; 66(03):246-250, discussion 250-251

12 Fleetwood IG, Embil JM, Ross IB. Nocardia asteroides cerebral abscess in immunocompetent hosts: report of three cases and review of surgical recommendations. Surg Neurol 2000;53(06): 605-610

13 Embil JM, Halliday WC, Nath A. Primary cerebellar T-cell lymphoma with acquired immunodeficiency syndrome. J Neurovirol 1997;3(03):229-232

14 Chen ST, Tang LM, Ro LS. Brain abscess as a complication of stroke. Stroke 1995;26(04):696-698

15 Börm W, Gleixner M. Nocardia brain abscess misinterpreted as cerebral infarction. J Clin Neurosci 2003;10(01):130-132 